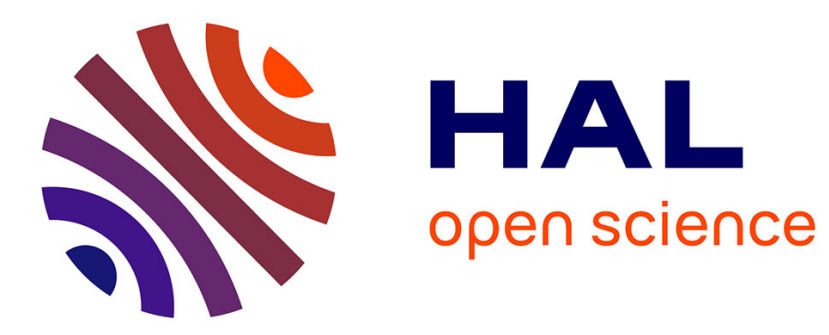

\title{
Local Euler-Maclaurin expansion of Barvinok valuations and Ehrhart coefficients of a rational polytope
}

\author{
Velleda Baldoni, Nicole Berline, Michèle Vergne
}

\section{To cite this version:}

Velleda Baldoni, Nicole Berline, Michèle Vergne. Local Euler-Maclaurin expansion of Barvinok valuations and Ehrhart coefficients of a rational polytope. Matthias Beck, Christian Haase, Bruce Reznick, Michèle Vergne, Volkmar Welker and Ruriko Yoshida. Integer Points in Polyhedra-Geometry, Number Theory, Representation Theory, Algebra, Optimization, Statistics, American Mathematical Society, pp.15-33, 2008, Contemporary Mathematics, Vol. 452, 978-0-8218-4173-0. 10.1090/conm/452 . hal00134312

\section{HAL Id: hal-00134312 \\ https://hal.science/hal-00134312}

Submitted on 1 Mar 2007

HAL is a multi-disciplinary open access archive for the deposit and dissemination of scientific research documents, whether they are published or not. The documents may come from teaching and research institutions in France or abroad, or from public or private research centers.
L'archive ouverte pluridisciplinaire HAL, est destinée au dépôt et à la diffusion de documents scientifiques de niveau recherche, publiés ou non, émanant des établissements d'enseignement et de recherche français ou étrangers, des laboratoires publics ou privés. 


\title{
Local Euler-Maclaurin expansion of Barvinok valuations and Ehrhart coefficients of a rational polytope
}

\author{
Velleda Baldoni, Nicole Berline and Michèle Vergne
}

December 2006

\section{Introduction}

Let $\mathfrak{p}$ be a rational polytope in $V=\mathbb{R}^{d}$ and $h(x)$ a polynomial function on $V$. A classical problem in Integer Programming is to compute the sum of values of $h(x)$ over the set of integral points of $\mathfrak{p}$,

$$
S(\mathfrak{p}, h)=\sum_{x \in \mathfrak{p} \cap \mathbb{Z}^{d}} h(x)
$$

When $\mathfrak{p}$ is dilated by an integer $n \in \mathbb{N}$, we obtain a function of $n$ which is quasi-polynomial, the so-called Ehrhart quasi-polynomial of the pair $(\mathfrak{p}, h)$

$$
S(n \mathfrak{p}, h)=\sum_{m=0}^{d+N} E_{m}(\mathfrak{p}, h, n) n^{m}
$$

of degree $d+N$ where $N=\operatorname{deg} h$. The coefficients $E_{m}(\mathfrak{p}, h, n)$ are periodic functions of $n \in \mathbb{N}$, with period the smallest integer $q$ such that $q \mathfrak{p}$ is a lattice polytope.

Replacing $h(x)$ by an exponential, we are led to study the analytic function on $V^{*}$ defined by

$$
S(\mathfrak{p})(\xi)=\sum_{x \in \mathfrak{p} \cap \mathbb{Z}^{d}} e^{\langle\xi, x\rangle} .
$$

If $\mathfrak{p}$ is any rational polyhedron, this sum still makes sense as a meromorphic function defined near 0 and the map $\mathfrak{p} \mapsto S(\mathfrak{p})(\xi)$ is a valuation. 
In [4], we proved that the meromorphic function $S(\mathfrak{p})(\xi)$ has a local EulerMaclaurin expansion

$$
S(\mathfrak{p})(\xi)=\sum_{\mathfrak{f}} \mu(\mathfrak{t}(\mathfrak{p}, \mathfrak{f}))(\xi) \int_{\mathfrak{f}} e^{\langle\xi, x\rangle} d x .
$$

The sum is taken over the set of faces $\mathfrak{f}$ of the polyhedron $\mathfrak{p}$. For each face $\mathfrak{f}$, the function $\mu(\mathfrak{t}(\mathfrak{p}, \mathfrak{f}))(\xi)$ is holomorphic near 0 , and it depends only on the transverse cone $\mathfrak{t}(\mathfrak{p}, \mathfrak{f})$ of $\mathfrak{p}$ along $\mathfrak{f}$. More precisely, once a rational scalar product is chosen on $V$, we define canonically a map $\mathfrak{a} \mapsto \mu(\mathfrak{a})$ from the set of rational affine cones $\mathfrak{a}$ in quotient spaces of $V$, with values in the space of functions on $V$ which are analytic near 0 , then we prove that these functions satisfy the above formula. The map $\mathfrak{a} \mapsto \mu(\mathfrak{a})$ is invariant under lattice translations, equivariant with respect to lattice preserving isometries, and it is a valuation on the set of affine cones with a fixed vertex (清, Theorems 17 and 18).

It is easy to see that the Ehrhart quasi-polynomial can be computed in terms of the Taylor coefficients of the functions $\mu(\mathfrak{t}(\mathfrak{p}, \mathfrak{f}))(\xi)$. For example, if $\mathfrak{p}$ is a lattice polytope and $h(x)=1$, we have ([四], Corollary 28)

$$
\operatorname{Card}\left(n \mathfrak{p} \cap \mathbb{Z}^{d}\right)=\sum_{\mathfrak{f}} \mu(\mathfrak{t}(\mathfrak{p}, \mathfrak{f}))(0) \operatorname{vol}(\mathfrak{f}) n^{\operatorname{dim} \mathfrak{f}} .
$$

Using the valuation property of $\mu(\mathfrak{a})$ and Barvinok's decomposition of a cone into unimodular cones, we thus obtained in [4] an algorithm for computing the Ehrhart quasi-polynomial. It has polynomial length with respect to the input $(\mathfrak{p}, h)$, when the dimension $d$ and the degree $N$ are fixed.

The valuation $S(\mathfrak{p}, h)$ has a natural generalization used by Barvinok in [2], the mixed valuation $S^{L}(\mathfrak{p}, h)$, where $L \subseteq V$ is a rational vector subspace. Denote the projected lattice on $V / L$ by $\Lambda_{L}$. For a polytope $\mathfrak{p} \subset V$ and a polynomial $h(x)$

$$
S^{L}(\mathfrak{p}, h)=\sum_{y \in \Lambda_{V / L}} \int_{\mathfrak{p} \cap(y+L)} h(x) d x .
$$

In other words, the polytope $\mathfrak{p}$ is sliced along lattice affine subspaces parallel to $L$ and the integrals of $h$ over the slices are added up. For $L=V$, there is only one term and $S^{V}(\mathfrak{p}, h)$ is just the integral of $h(x)$ over $\mathfrak{p}$, while, for $L=\{0\}$, we recover $S(\mathfrak{p}, h)$, the sum of values of $h(x)$ over the set of integral points of $\mathfrak{p}$. 
In the case $h(x)=1$, we write $S(\mathfrak{p})$ and $S^{L}(\mathfrak{p})$ in place of $S(\mathfrak{p}, 1)$ and $S^{L}(\mathfrak{p}, 1)$.

Using these mixed valuations, Barvinok gave an algorithm which computes the $r+1$ highest degree Ehrhart coefficients of $S(n \mathfrak{p})=\operatorname{Card}\left(n \mathfrak{p} \cap \mathbb{Z}^{d}\right)$, when $\mathfrak{p}$ is a simplex in $\mathbb{R}^{d}$. Barvinok's algorithm has polynomial length when $d$ is an input, provided $r$ is fixed. The method consists in reducing the problem to summations over lattice points in dimension $\leq r$.

Barvinok considers particular linear combinations

$$
\sum_{L \in \mathcal{L}} \rho(L) S^{L}(\mathfrak{p})
$$

where $\mathcal{L}$ is a finite set of rational vector subspaces of $V$ which is closed under sum, and the coefficients $\rho(L)$ are integers which satisfy the following relation between characteristic functions:

$$
\chi\left(\cup_{L \in \mathcal{L}} L^{\perp}\right)=\sum_{L \in \mathcal{L}} \rho(L) \chi\left(L^{\perp}\right),
$$

where $L^{\perp} \subseteq V^{*}$ is the orthogonal of $L$. We call a function $\mathcal{L} \rightarrow \mathbb{Z}$ with this property a patchwork function on $\mathcal{L}$.

When $\mathfrak{p}$ is dilated by an integer $n, S^{L}(n \mathfrak{p})$ is again given by a quasipolynomial in $n$, as is a linear combination

$$
\sum_{L \in \mathcal{L}} \rho(L) S^{L}(n \mathfrak{p})=\sum_{m=0}^{d} \nu_{m}(\mathfrak{p}, n) n^{m} .
$$

The main theoretical result of [2], Theorem (1.3), is the following: if $\mathcal{L}$ is a family of subspaces which is closed under sum and contains the vector subspace lin $(\mathfrak{f})$ parallel to $\mathfrak{f}$, for every face $\mathfrak{f}$ of codimension $\leq r$ of $\mathfrak{p}$, and if $\rho$ is a patchwork function on $\mathcal{L}$, then the $r+1$ highest degree coefficients $\nu_{m}(\mathfrak{p}, n)$, for $m=d, \ldots, d-r$, are equal to the corresponding Ehrhart coefficients of $S(n \mathfrak{p})=\operatorname{Card}\left(n \mathfrak{p} \cap \mathbb{Z}^{d}\right)$.

In the present article, we introduce the meromorphic functions which extend $S^{L}(\mathfrak{p})$. For any polyhedron $\mathfrak{p}$,

$$
S^{L}(\mathfrak{p})(\xi)=\sum_{y \in \Lambda_{V / L}} \int_{\mathfrak{p} \cap(y+L)} e^{\langle\xi, x\rangle} d x
$$


is defined as a meromorphic function near $\xi=0$. We show that $S^{L}(\mathfrak{p})(\xi)$ also enjoys a local Euler-Maclaurin expansion (Theorem 8),

$$
S^{L}(\mathfrak{p})(\xi)=\sum_{\mathfrak{f}} \mu^{L}(\mathfrak{t}(\mathfrak{p}, \mathfrak{f}))(\xi) \int_{\mathfrak{f}} e^{\langle\xi, x\rangle} d x .
$$

Furthermore, for a linear combination of Barvinok type, if $\mathfrak{f}$ is a face of $\mathfrak{p}$ such that $\operatorname{lin}(\mathfrak{f}) \in \mathcal{L}$, we prove that the $\mathfrak{f}$-term in the Euler-Maclaurin expansions of

$$
S^{\mathcal{L}, \rho}(\mathfrak{p})(\xi)=\sum_{L \in \mathcal{L}} \rho(L) S^{L}(\mathfrak{p})(\xi)
$$

and of the usual valuation $S(\mathfrak{p})(\xi)$ are equal (Theorem 17):

$$
\sum_{L \in \mathcal{L}} \rho(L) \mu^{L}(\mathfrak{t}(\mathfrak{p}, \mathfrak{f}))(\xi)=\mu(\mathfrak{t}(\mathfrak{p}, \mathfrak{f}))(\xi) .
$$

This is the main result of the present article. From the relation between Ehrhart quasi-polynomials and Euler-Maclaurin expansions, it implies Barvinok's Theorem (1.3).

Actually, we derive from Theorem 17 another computation of the $r+1$ highest coefficients of the Ehrhart quasi-polynomial for a pair $(\mathfrak{p}, h)$, based on Brion's decomposition of a polytope into cones, in the line of [1] and [6].

For each vertex $s$ of $\mathfrak{p}$, let $\mathfrak{c}_{s}$ be the cone of feasible directions of $\mathfrak{p}$ at $s$. Instead of the full family $\mathcal{L}$ generated by taking sums of the subspaces $\operatorname{lin}(\mathfrak{f})$, when $\mathfrak{f}$ runs over the set of faces of codimension $\leq r$ of the polytope $\mathfrak{p}$, we consider, for each vertex $s$ of $\mathfrak{p}$, the family $\mathcal{L}_{s}$ generated by faces of $\mathfrak{c}_{s}$ of codimension $\leq r$. The point in taking a family which depends on $s$ lies in the case where $\mathfrak{p}$ is simplicial. Then $\mathcal{L}_{s}$ consists only of the spaces $\operatorname{lin}(\mathfrak{f})$ where $\mathfrak{f}$ is a face of $\mathfrak{c}_{s}$ of codimension $\leq r$, as this set is already closed under sum. Moreover the coefficients $\rho(L)$ are just signed binomial coefficients (Lemma 15), and the computation of $S^{L}\left(\mathfrak{c}_{s}\right)$ is easier when $L$ is parallel to a face of $\mathfrak{c}_{s}$ (Example 6).

Let us describe our method in the simpler case of a lattice polytope $\mathfrak{p}$ and polynomial $h(x)=1$. By Brion's theorem, we have

$$
S(\mathfrak{p})(\xi)=\sum_{s} e^{\langle\xi, s\rangle} S\left(\mathfrak{c}_{s}\right)(\xi) .
$$

For each vertex $s$, let $\rho_{s}: \mathcal{L}_{s} \rightarrow \mathbb{Z}$ be a patchwork function. We define

$$
\mathcal{B}(\mathfrak{p})(\xi)=\sum_{s} e^{\langle\xi, s\rangle} S^{\mathcal{L}_{s}, \rho_{s}}\left(\mathfrak{c}_{s}\right)(\xi)
$$


For the dilated polytope $n \mathfrak{p}$, we have

$$
S(n \mathfrak{p})(\xi)=\sum_{s} e^{n\langle\xi, s\rangle} S\left(\mathfrak{c}_{s}\right)(\xi)=\sum_{m \geq 0} \frac{n^{m}}{m !} \sum_{s}\langle\xi, s\rangle^{m} S\left(\mathfrak{c}_{s}\right)(\xi) .
$$

Hence, the meromorphic function $\frac{1}{m !} \sum_{s}\langle\xi, s\rangle^{m} S\left(\mathfrak{c}_{s}\right)(\xi)$ is actually regular at $\xi=0$ and its value at $\xi=0$ is the $m$ th Ehrhart coefficient of $\mathfrak{p}$.

We have similarly

$$
\mathcal{B}(n \mathfrak{p})(\xi)=\sum_{m \geq 0} \frac{n^{m}}{m !} \sum_{s}\langle\xi, s\rangle^{m} S^{\mathcal{L}_{s}, \rho_{s}}\left(\mathfrak{c}_{s}\right)(\xi) .
$$

The meromorphic functions $S^{L}\left(\mathfrak{c}_{s}\right)(\xi)$ and $S^{\mathcal{L}_{s}, \rho_{s}}\left(\mathfrak{c}_{s}\right)(\xi)$ have a special form: they can be written as the quotient of an analytic function by a product of $d^{\prime} \leq d$ linear forms. Such a function $\phi$ has an expansion into rational functions $\phi=\sum_{j>-d} \phi_{[j]}$ where $\phi_{[j]}$ is homogeneous of total degree $j$.

Now it follows from our main theorem that, for $m \geq d-r$, we have

$$
S\left(\mathfrak{c}_{s}\right)_{[-m]}(\xi)=S^{\mathcal{L}_{s}, \rho_{s}}\left(\mathfrak{c}_{s}\right)_{[-m]}(\xi),
$$

hence the zero degree terms of $\sum_{s}\langle\xi, s\rangle^{m} S\left(\mathfrak{c}_{s}\right)(\xi)$ and $\sum_{s}\langle\xi, s\rangle^{m} S^{\mathcal{L}_{s}, \rho_{s}}\left(\mathfrak{c}_{s}\right)(\xi)$ are equal. Therefore the latter is also analytic at $\xi=0$ and its value at $\xi=0$ is the $m$ th Ehrhart coefficient of $\mathfrak{p}$. This is the content of Theorem 20.

Thus, besides taking care of any polynomial $h(x)$, not only $h(x)=1$, this method to compute the $r+1$ highest degree Ehrhart coefficients for the pair $(\mathfrak{p}, h)$ leads to a simpler algorithm than the one proposed in [2]. When $\mathfrak{p}$ is a rational simplex, the contributions of the terms of the form $S^{L}\left(\mathfrak{c}_{s}\right)(\xi)$ when $L \in \mathcal{L}_{s}$ are immediately reduced to the computation of a function $S(\mathfrak{a})$ with $\mathfrak{a}$ a simplicial cone of dimension smaller or equal to $r$.

There is also another possible implementation of an algorithm to compute the $r+1$ Ehrhart highest degree coefficients for the pair $(\mathfrak{p}, h)$ based on the results of [1. As seen from Equation (1), this involves the computation of the analytic function $\mu(\mathfrak{t}(\mathfrak{p}, \mathfrak{f}))$, also associated to simplicial cones in dimension smaller or equal to $r$. We plan to compare the implementation of both methods in the near future. 


\section{Local Euler-Maclaurin expansion of a mixed valuation $S^{L}$}

We consider a rational vector space $V$ of dimension $d$, that is to say a finite dimensional real vector space with a lattice denoted by $\Lambda_{V}$ or simply $\Lambda$. We will need to consider subspaces and quotient spaces of $V$, this is why we cannot just let $V=\mathbb{R}^{d}$ and $\Lambda=\mathbb{Z}^{d}$. By lattice, we mean a discrete additive subgroup of $V$ which generates $V$ as a vector space. Hence, a lattice is generated by a basis of the vector space $V$. A basis of $V$ which is a $\mathbb{Z}$-basis of $\Lambda_{V}$ is called an integral basis. The elements of $\Lambda$ are called integral. An element $x \in V$ is called rational if $q x \in \Lambda$ for some integer $q \neq 0$. The space of rational points in $V$ is denoted by $V_{\mathbb{Q}}$. A subspace $L$ of $V$ is called rational if $L \cap \Lambda$ is a lattice in $L$. If $L$ is a rational subspace, the image of $\Lambda$ in $V / L$ is a lattice in $V / L$, so that $V / L$ is a rational vector space. We will call the image of $\Lambda$ in $V / L$ the projected lattice.

Example 1 Let $V=\mathbb{R}^{2}$ with standard lattice $\mathbb{Z}^{2}$. Let $v_{1}, v_{2}$ be two primitive integral independent vectors. Using an integral basis with first basis vector $v_{1}$, a straightforward computation shows that the projected lattice on $\mathbb{R}^{2} / \mathbb{R} v_{1}$ is $\mathbb{Z} \frac{\bar{v}_{2}}{\operatorname{det}\left(v_{1}, v_{2}\right)}$, where $\bar{v}_{2}$ is the projection of $v_{2}$ on $\mathbb{R}^{2} / \mathbb{R} v_{1}$.

A rational space $V$, with lattice $\Lambda$, has a canonical Lebesgue measure, for which $V / \Lambda$ has measure 1. An affine subspace $L$ of $V$ is called rational if it is a translate of a rational subspace by a rational element. It is similarly provided with a canonical Lebesgue measure. We will denote this measure by $d m_{L}$.

We will denote elements of $V$ by latin letters $x, y, v, \ldots$ and elements of $V^{*}$ by greek letters $\xi, \alpha, \ldots$ We denote the duality bracket by $\langle\xi, x\rangle$.

If $S$ is a subset of $V$, we denote by $\langle S\rangle$ the affine subspace generated by $S$. If $S$ consists of rational points, then $\langle S\rangle$ is rational. Remark that $\langle S\rangle$ may contain no integral point. We denote by $\operatorname{lin}(S)$ the vector subspace of $V$ parallel to $\langle S\rangle$. $S$ :

If $S$ is a subset of $V$, we denote by $S^{\perp}$ the subspace of $V^{*}$ orthogonal to

$$
S^{\perp}=\left\{\xi \in V^{*} ;\langle\xi, x\rangle=0 \text { for all } x \in S\right\} .
$$

If $L$ is a subspace of $V$, the dual space $(V / L)^{*}$ is canonically identified with the subspace $L^{\perp} \subset V^{*}$. 
A convex rational polyhedron $\mathfrak{p}$ in $V$ (we will simply say polyhedron) is, by definition, the intersection of a finite number of half spaces bounded by rational affine hyperplanes. We say that $\mathfrak{p}$ is solid (in $\mathrm{V}$ ) if $\langle\mathfrak{p}\rangle=V$. A polytope $\mathfrak{p}$ is a compact polyhedron.

The set of non negative real numbers is denoted by $\mathbb{R}_{+}$. A convex rational cone $\mathfrak{c}$ in $V$ is a closed convex cone $\sum_{i=1}^{k} \mathbb{R}_{+} v_{i}$ which is generated by a finite number of elements $v_{i}$ of $V_{\mathbb{Q}}$. In this article, we simply say cone instead of convex rational cone.

An affine (rational) cone $\mathfrak{a}$ is, by definition, the translate of a cone in $V$ by a rational element $s \in V_{\mathbb{Q}}$. This cone is uniquely defined by $\mathfrak{a}$.

A cone $\mathfrak{c}$ is called simplicial if it is generated by independent elements of $V_{\mathbb{Q}}$. A simplicial cone $\mathfrak{c}$ is called unimodular if it is generated by independent integral vectors $v_{1}, \ldots, v_{k}$ such that $\left\{v_{1}, \ldots, v_{k}\right\}$ can be completed in an integral basis of $V$. An affine cone $\mathfrak{a}$ is called simplicial (resp. simplicial unimodular) if it is the translate of a simplicial (resp. simplicial unimodular) cone.

An affine cone $\mathfrak{a}$ is called pointed if it does not contain any straight line.

The set of faces of an affine cone $\mathfrak{a}$ is denoted by $\mathcal{F}(\mathfrak{a})$. If $\mathfrak{a}$ is pointed, then the vertex of $\mathfrak{a}$ is the unique face of dimension 0 , while $\mathfrak{a}$ is the unique face of maximal dimension $\operatorname{dim} \mathfrak{a}$.

Let us recall the definition of the transverse cone $\mathfrak{t}(\mathfrak{p}, \mathfrak{f})$ of a polyhedron $\mathfrak{p}$ along one of its faces $\mathfrak{f}$. Let $x$ be a point in the relative interior of $\mathfrak{f}$. The cone of feasible directions of $\mathfrak{p}$ at $x$ is the set $\mathfrak{c}(\mathfrak{p}, \mathfrak{f}):=\{v \in V ; x+\epsilon v \in$ $\mathfrak{p}$ for $\epsilon>0$ small enough $\}$. It does not depend on the choice of $x$. We denote the projection $V \rightarrow V / \operatorname{lin}(\mathfrak{f})$ by $\pi_{\mathfrak{f}}$. Then $\mathfrak{t}(\mathfrak{p}, \mathfrak{f})$ is the image $\pi_{\mathfrak{f}}(\mathfrak{f}+\mathfrak{c}(\mathfrak{p}, \mathfrak{f}))$ of the affine cone $\mathfrak{f}+\mathfrak{c}(\mathfrak{p}, \mathfrak{f})$ in $V / \operatorname{lin}(\mathfrak{f})$. It is a solid pointed affine cone in the quotient space $V / \operatorname{lin}(\mathfrak{f})$ with vertex $\pi_{\mathfrak{f}}(<\mathfrak{f}>)$. In particular, if $v$ is a vertex of $\mathfrak{p}$, the transverse cone $\mathfrak{t}(\mathfrak{p}, v)$ coincides with the supporting cone $v+\mathfrak{c}(\mathfrak{p}, v) \subset V$.

If $\mathfrak{a}$ is an affine cone and $\mathfrak{f}$ is a face of $\mathfrak{a}$, then $\mathfrak{c}(\mathfrak{a}, \mathfrak{f})=\mathfrak{a}+\operatorname{lin}(\mathfrak{f})$ and the transverse cone $\mathfrak{t}(\mathfrak{a}, \mathfrak{f})$ of $\mathfrak{a}$ along $\mathfrak{f}$ is just the projection $\pi_{\mathfrak{f}}(\mathfrak{a})$ of $\mathfrak{a}$ on $V / \operatorname{lin}(\mathfrak{f})$.

Definition 2 Denote by $\mathcal{H}\left(V^{*}\right)$ the ring of analytic functions around $0 \in V^{*}$. Denote by $\mathcal{M}\left(V^{*}\right)$ the ring of meromorphic functions defined around $0 \in$ $V^{*}$ and by $\mathcal{M}_{\ell}\left(V^{*}\right) \subset \mathcal{M}\left(V^{*}\right)$ the subring consisting of those meromorphic functions $\phi(\xi)$ such that there exists a product of linear forms $D(\xi)$ with

$$
D(\xi) \phi(\xi) \in \mathcal{H}\left(V^{*}\right) \text {. }
$$


A function $\phi(\xi) \in \mathcal{M}_{\ell}\left(V^{*}\right)$ has a unique expansion into homogeneous rational functions

$$
\phi(\xi)=\sum_{m>>-\infty} \phi_{[m]}(\xi)
$$

where $m$ is the total degree.

If $P$ is a homogeneous polynomial on $V^{*}$ of degree $p$, and $D$ a product of $r$ linear forms, then $\frac{P}{D}$ is an element in $\mathcal{M}_{\ell}\left(V^{*}\right)$ homogeneous of degree $m=p-r$.

Let us recall the definition of the function $I(\mathfrak{p}) \in \mathcal{M}_{\ell}\left(V^{*}\right)$ associated to a polyhedron $\mathfrak{p}$, (see for instance the survey [3]).

Proposition 3 There exists a map I which to every polyhedron $\mathfrak{p} \subset V$ associates a meromorphic function with rational coefficients $I(\mathfrak{p}) \in \mathcal{M}_{\ell}\left(V^{*}\right)$, so that the following properties hold:

(a) If $\mathfrak{p}$ contains a straight line, then $I(\mathfrak{p})=0$.

(b) If $\xi \in V^{*}$ is such that $\left|e^{\langle\xi, x\rangle}\right|$ is integrable over $\mathfrak{p}$, then

$$
I(\mathfrak{p})(\xi)=\int_{\mathfrak{p}} e^{\langle\xi, x\rangle} d m_{<\mathfrak{p}\rangle}(x) .
$$

(c) For every point $s \in V_{\mathbb{Q}}$, we have

$$
I(s+\mathfrak{p})(\xi)=e^{\langle\xi, s\rangle} I(\mathfrak{p})(\xi) .
$$

(d) The map I is a simple valuation: if the characteristic functions $\chi\left(\mathfrak{p}_{i}\right)$ of a family of polyhedra $\mathfrak{p}_{i}$ satisfy a linear relation $\sum_{i} r_{i} \chi\left(\mathfrak{p}_{i}\right)=0$, then the functions $I\left(\mathfrak{p}_{i}\right)$ satisfy the relation

$$
\sum_{\left\{i,<\mathfrak{p}_{i}>=V\right\}} r_{i} I\left(\mathfrak{p}_{i}\right)=0
$$

In the following proposition, we define the mixed valuation $\mathfrak{p} \mapsto S^{L}(\mathfrak{p})$ associated to a rational vector subspace $L \subseteq V$. To any polyhedron $\mathfrak{p}$, we associate a meromorphic function $S^{L}(\mathfrak{p})(\xi) \in \mathcal{M}\left(V^{*}\right)$. If $\mathfrak{p}$ is compact, this function is actually regular at 0 , and its value for $\xi=0$ is the valuation $E_{L^{\perp}}(\mathfrak{p})$ considered by Barvinok [2].

We denote by $\Lambda_{V / L}$ the projection on $V / L$ of the lattice $\Lambda$. 
Proposition 4 Let $L \subseteq V$ be a rational subspace. There exists a map $S^{L}$ which to every rational polyhedron $\mathfrak{p} \subset V$ associates a meromorphic function with rational coefficients $S^{L}(\mathfrak{p}) \in \mathcal{M}\left(V^{*}\right)$ so that the following properties hold:

(a) If $\mathfrak{p}$ contains a line, then $S^{L}(\mathfrak{p})=0$.

(b)

$$
S^{L}(\mathfrak{p})(\xi)=\sum_{y \in \Lambda_{V / L}} \int_{\mathfrak{p} \cap(y+L)} e^{\langle\xi, x\rangle} d m_{L}(x),
$$

for every $\xi \in V^{*}$ such that the above sum converges.

(c) For every point $s \in \Lambda$, we have

$$
S^{L}(s+\mathfrak{p})(\xi)=e^{\langle\xi, s\rangle} S^{L}(\mathfrak{p})(\xi) .
$$

(d) The map $S^{L}$ is a valuation: if the characteristic functions $\chi\left(\mathfrak{p}_{i}\right)$ of a family of polyhedra $\mathfrak{p}_{i}$ satisfy a linear relation $\sum_{i} r_{i} \chi\left(\mathfrak{p}_{i}\right)=0$, then the functions $S^{L}\left(\mathfrak{p}_{i}\right)$ satisfy the same relation

$$
\sum_{i} r_{i} S^{L}\left(\mathfrak{p}_{i}\right)=0
$$

For $L=\{0\}$, we recover the valuation $S$ given by

$$
S(\mathfrak{p})(\xi)=\sum_{x \in \mathfrak{p} \cap \Lambda} e^{\langle\xi, s\rangle}
$$

provided this sum is convergent.

For $L=V$, we have $S^{V}(\mathfrak{p})=I(\mathfrak{p})$, if $\mathfrak{p}$ is solid, and $S^{V}(\mathfrak{p})=0$ otherwise.

The proof is entirely analogous to the case $L=\{0\}$, see Theorem 3.1 in [3], and we omit it.

Remark 5 The function $S^{L}(\mathfrak{p})$ is actually an element of $\mathcal{M}_{\ell}\left(V^{*}\right)$, but we do not prove it at this point. Let $\mathfrak{a}$ be an affine cone and $\left\{v_{i}\right\}$ the generators of its edges. It will follow from the Euler-Maclaurin expansion of $S^{L}(\mathfrak{a})$ (Theorem 8) that $\prod_{i}\left\langle\xi, v_{i}\right\rangle S^{L}(\mathfrak{a})(\xi)$ is analytic near zero for any $L$. It would be interesting to prove it a priori. By Brion's theorem and the valuation property, it follows in particular that $S^{L}(\mathfrak{p}) \in \mathcal{M}_{\ell}\left(V^{*}\right)$. 
Example 6 Let $\mathfrak{a}$ be a simplicial affine cone in the space $V$, and assume that $L=\operatorname{lin}\left(\mathfrak{f}_{1}\right)$ for some face $\mathfrak{f}_{1}$ of $\mathfrak{a}$. In this case, $S^{L}(\mathfrak{a})(\xi)$ decomposes as product of an integral and a discrete sum. For simplicity, assume that $\mathfrak{a}$ is solid. Let $\mathfrak{f}_{2}$ be the face of $\mathfrak{a}$ such $V=\operatorname{lin}\left(\mathfrak{f}_{1}\right) \oplus \operatorname{lin}\left(\mathfrak{f}_{2}\right)$. We write $x=x_{1}+x_{2}$ and $\xi=\xi_{1}+\xi_{2}$ for the corresponding decompositions of $x \in V$ and $\xi \in V^{*}$. Then $\mathfrak{a}=\mathfrak{a}_{1}+\mathfrak{a}_{2}$ where $\mathfrak{a}_{i}$ is a simplicial affine cone in $\operatorname{lin}\left(\mathfrak{f}_{i}\right)$. Let us denote by $\Lambda_{2}$ the projected lattice in $V / \operatorname{lin}\left(\mathfrak{f}_{1}\right) \sim \operatorname{lin}\left(\mathfrak{f}_{2}\right)$. From (2), we obtain immediately

$$
S^{L}(\mathfrak{a})\left(\xi_{1}+\xi_{2}\right)=I\left(\mathfrak{a}_{1}\right)\left(\xi_{1}\right) \sum_{x_{2} \in \mathfrak{a}_{2} \cap \Lambda_{2}} e^{\left\langle\xi_{2}, x_{2}\right\rangle}
$$

Notice that the lattice $\Lambda_{2}$ is usually bigger than $\Lambda \cap \operatorname{lin}\left(\mathfrak{f}_{2}\right)$.

Example 7 Let $V=\mathbb{R}^{2}$ with the standard lattice. We compute $S^{L}(\mathfrak{a})$ when $\mathfrak{a}$ is a cone and $L$ is a line. Let $\mathfrak{a}=\mathbb{R}_{+} v_{1}+\mathbb{R}_{+} v_{2}$, where $v_{1}, v_{2}$ are two linearly independent primitive integral vectors.

(a) Assume that $L$ is the line supporting an edge of $\mathfrak{a}$, say $L=\mathbb{R} v_{1}$. We identify $V / L$ to $\mathbb{R} v_{2}$. The projected lattice is $\Lambda_{2}=\mathbb{Z} \frac{v_{2}}{\operatorname{det}\left(v_{1}, v_{2}\right)}$, (Example 团), hence, by (3) in Example 6, we have

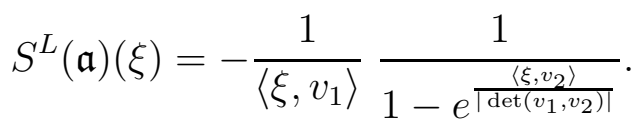

(b) Assume now that $L$ is transverse to both edges of $\mathfrak{a}$. Assume that $\operatorname{det}\left(v_{1}, v_{2}\right)>0$. Let $L=\mathbb{R} u$ where $u$ is a primitive integral vector chosen so that $\operatorname{det}\left(u, v_{2}\right)>0$. Let $\mathfrak{a}_{i}=\mathbb{R}_{+} u+\mathbb{R}_{+} v_{i}$ for $i=1,2$. We decompose the characteristic function of the cone $\mathfrak{a}$ as $\chi(\mathfrak{a})=\chi\left(\mathfrak{a}_{2}\right)+\chi\left(\mathfrak{a}_{1}\right)-\chi\left(\mathbb{R}_{+} u\right)$ or $\chi(\mathfrak{a})=\chi\left(\mathfrak{a}_{2}\right)-\chi\left(\mathfrak{a}_{1}\right)+\chi\left(\mathbb{R}_{+} v_{1}\right)$, depending on whether $u$ belongs to $\mathfrak{a}$ or not. Using the valuation property, case (a) and the relation

$$
\frac{1}{1-e^{x}}+\frac{1}{1-e^{-x}}=1
$$

we obtain in both cases

$$
S^{L}(\mathfrak{a})(\xi)=-\frac{1}{\langle\xi, u\rangle}\left(\frac{1}{1-e^{\frac{\left\langle\xi, v_{2}\right\rangle}{\operatorname{det}\left(u, v_{2}\right)}}}-\frac{1}{1-e^{\frac{\left\langle\xi, v_{1}\right\rangle}{\operatorname{det}\left(u, v_{1}\right)}}}\right) .
$$

In this example, we see that $\left\langle\xi, v_{1}\right\rangle\left\langle\xi, v_{2}\right\rangle S^{L}(\mathfrak{a})(\xi)$ is indeed analytic near $\xi=0$. 
In the following theorem and its applications, we will consider the functions $S^{L}(\mathfrak{p})$ when the space $V$ is replaced with a quotient space $W$. We denote by $\mathcal{C}(W)$ the set of affine cones in $W$. Thus if $\mathfrak{a} \in \mathcal{C}(W)$, and $L$ a rational subspace of $W$, the function $S^{L}(\mathfrak{a})$ is a meromorphic function on $W^{*}$. We are going to show that the function $S^{L}(\mathfrak{a})$ has a local Euler-Maclaurin expansion, which generalizes the case $L=\{0\}$ of $[4]$.

Theorem 8 Let $V$ be a rational space and $Q$ a rational scalar product on $V^{*}$. There exists a unique family of maps $\mu_{W}^{L}$, indexed by pairs $(W, L)$ where $W$ is a rational quotient space of $V$ and $L$ is a rational vector subspace of $W$ such that the family enjoys the following properties:

(a) $\mu_{W}^{L}$ maps $\mathcal{C}(W)$ to $\mathcal{H}\left(W^{*}\right)$, the space of analytic functions on $W^{*}$.

(b) If $W=\{0\}$, then $\mu_{\{0\}}^{\{0\}}(\{0\})=1$.

(c) For $\operatorname{dim} W>0$ and $L=W$, then $\mu_{W}^{W}(\mathfrak{a})=0$.

(d) If the affine cone $\mathfrak{a} \in \mathcal{C}(W)$ contains a straight line, then $\mu_{W}^{L}(\mathfrak{a})=0$.

(e) For any affine cone $\mathfrak{a}$ in $W$, the following formula holds

$$
S^{L}(\mathfrak{a})=\sum_{\mathfrak{f} \in \mathcal{F}(\mathfrak{a})} \mu_{W / \operatorname{lin}(\mathfrak{f})}^{L+\operatorname{lin}(\mathfrak{f}) / \operatorname{lin}(\mathfrak{f})}(\mathfrak{t}(\mathfrak{a}, \mathfrak{f})) I(\mathfrak{f})
$$

where the sum is over all faces of the cone $\mathfrak{a}$.

In this last formula, the function $\mu_{W / \operatorname{lin}(\mathfrak{f})}^{L+\operatorname{lin}(\mathfrak{f}) / \operatorname{lin}(\mathfrak{f})}(\mathfrak{t}(\mathfrak{a}, \mathfrak{f}))$ is considered as a function on $W^{*}$ itself by means of the orthogonal projection $W^{*} \rightarrow(W / \operatorname{lin}(\mathfrak{f}))^{*}=$ $(\operatorname{lin}(\mathfrak{f}))^{\perp}$ with respect to the scalar product on $W^{*} \subset V^{*}$.

Proof. The proof is entirely similar to the case $L=\{0\}$ studied in [1]. Note that $\mu_{W}^{\{0\}}$ coincides with the map denoted by $\mu_{W}$ in $\llbracket$. The only new item is (c). It follows immediately from the relation $S^{W}(\mathfrak{a})=I(\mathfrak{a})$.

Remark 9 Let $\mathfrak{a}$ be a solid cone in $W$, and let $\mathfrak{f}$ be a face of $\mathfrak{a}$ such that $\operatorname{dim} \mathfrak{f}<\operatorname{dim} W$. If $L$ is transverse to the face $\mathfrak{f}$, that is, if $L+\operatorname{lin}(\mathfrak{f})=W$, then $\mu_{W / \operatorname{lin}(\mathfrak{f})}^{L+\operatorname{lin}(\mathfrak{l i n}(\mathfrak{f})}(\mathfrak{t}(\mathfrak{a}, \mathfrak{f}))=0$. This follows from $(c)$.

From now on we omit the subscript $W$, thus we write $\mu^{L}$ in place of $\mu_{W}^{L}$. The next theorem and its proof are also entirely similar to the case $L=\{0\}$ in [4]. 
Theorem 10 The analytic functions defined in Theorem \& have the following properties:

(a) For any $x \in \Lambda$, one has $\mu^{L}(x+\mathfrak{a})=\mu^{L}(\mathfrak{a})$.

(b) The map $(\mathfrak{a}, L) \mapsto \mu^{L}(\mathfrak{a})$ is equivariant with respect to lattice-preserving isometries. In other words, let $g$ be an isometry of $W$ which preserves the lattice $\Lambda_{W}$. Then $\mu^{g(L)}(g(\mathfrak{a}))\left({ }^{t} g^{-1} \xi\right)=\mu^{L}(\mathfrak{a})(\xi)$.

(c) If $W$ is an orthogonal sum $W=W_{1} \oplus W_{2}$ of rational spaces, $L_{i} \subseteq W_{i}$ and $\mathfrak{a}_{i}$ is an affine cone in $W_{i}$ for $i=1,2$, then

$$
\mu^{L_{1} \oplus L_{2}}\left(\mathfrak{a}_{1}+\mathfrak{a}_{2}\right)=\mu^{L_{1}}\left(\mathfrak{a}_{1}\right) \mu^{L_{2}}\left(\mathfrak{a}_{2}\right) .
$$

(d) For a fixed $s \in W_{\mathbb{Q}}$, the map $\mathfrak{c} \rightarrow \mu^{L}(s+\mathfrak{c})$ is a valuation on the set of cones in $W$.

(e) Let $\mathfrak{p} \subset W$ be a rational polyhedron, then

$$
S^{L}(\mathfrak{p})=\sum_{\mathfrak{f} \in \mathcal{F}(\mathfrak{p})} \mu^{L+\operatorname{lin}(\mathfrak{f}) / \operatorname{lin}(\mathfrak{f})}(\mathfrak{t}(\mathfrak{p}, \mathfrak{f})) I(\mathfrak{f}) .
$$

Example 11 Let us compute the function $\mu^{L}$ for the various transverse

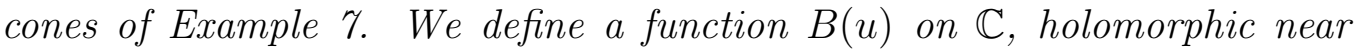
0, by

We have

$$
B(u)=\frac{1}{1-e^{u}}+\frac{1}{u}
$$

$$
I(\mathfrak{a})(\xi)=\frac{\left|\operatorname{det}\left(v_{1}, v_{2}\right)\right|}{\left\langle\xi, v_{1}\right\rangle\left\langle\xi, v_{2}\right\rangle} .
$$

Consider case (b) where $L$ is transverse to both edges $\mathfrak{f}_{i}=\mathbb{R}_{+} v_{i}$ of $\mathfrak{a}$.

Using the equation $\operatorname{det}\left(v_{1}, v_{2}\right) u=\operatorname{det}\left(u, v_{2}\right) v_{1}-\operatorname{det}\left(u, v_{1}\right) v_{2}$, we have

$$
I(\mathfrak{a})(\xi)=\frac{1}{\langle\xi, u\rangle}\left(\frac{\operatorname{det}\left(u, v_{2}\right)}{\left\langle\xi, v_{2}\right\rangle}-\frac{\operatorname{det}\left(u, v_{1}\right)}{\left\langle\xi, v_{1}\right\rangle}\right) .
$$

Thus we can rewrite (5) as

$$
S^{L}(\mathfrak{a})=\mu^{L}(\mathfrak{a})+\sum_{i=1,2} \mu^{L+\operatorname{lin}\left(\mathfrak{f}_{i}\right) / \operatorname{lin}\left(\mathfrak{f}_{i}\right)}\left(\left(\mathfrak{t}\left(\mathfrak{a}, \mathfrak{f}_{i}\right)\right) I\left(\mathfrak{f}_{i}\right)+I(\mathfrak{a}),\right.
$$

with

$$
\begin{aligned}
& \mu^{L}(\mathfrak{a})(\xi)=\frac{1}{\langle\xi, u\rangle}\left[B\left(\frac{\left\langle\xi, v_{1}\right\rangle}{\operatorname{det}\left(u, v_{1}\right)}\right)-B\left(\frac{\left\langle\xi, v_{2}\right\rangle}{\operatorname{det}\left(u, v_{2}\right)}\right)\right], \\
& \mu^{L+\operatorname{lin}\left(\mathfrak{f}_{i}\right) / \operatorname{lin}\left(\mathfrak{f}_{i}\right)}\left(\left(\mathfrak{t}\left(\mathfrak{a}, \mathfrak{f}_{i}\right)\right)=0 \text { for } i=1,2,\right.
\end{aligned}
$$


Observe that (17) is indeed regular at $\xi=0$.

In case (a) where $L=\mathbb{R} v_{1}$, we have $L+\operatorname{lin}\left(\mathfrak{f}_{1}\right) / \operatorname{lin}\left(\mathfrak{f}_{1}\right)=\{0\}$. Let us assume that $\operatorname{det}\left(v_{1}, v_{2}\right)>0$. Then we have, by [4],

$$
\mu^{\{0\}}\left(\mathfrak{t}\left(\mathfrak{a}, \mathfrak{f}_{1}\right)\right)(\xi)=B\left(\frac{-C_{1}\left\langle\xi, v_{1}\right\rangle+\left\langle\xi, v_{2}\right\rangle}{\operatorname{det}\left(v_{1}, v_{2}\right)}\right)
$$

with $C_{1}=\frac{Q\left(v_{1}, v_{2}\right)}{Q\left(v_{1}, v_{1}\right)}$.

As $I\left(\mathfrak{f}_{1}\right)(\xi)=-\frac{1}{\left\langle\xi, v_{1}\right\rangle}$, we can rewrite (团) as

$$
S^{L}(\mathfrak{a})=\mu^{L}(\mathfrak{a})+\mu^{\{0\}}\left(\mathfrak{t}\left(\mathfrak{a}, \mathfrak{f}_{1}\right)\right) I\left(\mathfrak{f}_{1}\right)+\mu^{L+\operatorname{lin}\left(\mathfrak{f}_{2}\right) / \operatorname{lin}\left(\mathfrak{f}_{2}\right)}\left(\left(\mathfrak{t}\left(\mathfrak{a}, \mathfrak{f}_{2}\right)\right) I\left(\mathfrak{f}_{2}\right)+I(\mathfrak{a}),\right.
$$

with

$$
\mu^{L}(\mathfrak{a})(\xi)=\frac{1}{\left\langle\xi, v_{1}\right\rangle}\left[B\left(\frac{-C_{1}\left\langle\xi, v_{1}\right\rangle+\left\langle\xi, v_{2}\right\rangle}{\operatorname{det}\left(v_{1}, v_{2}\right)}\right)-B\left(\frac{\left\langle\xi, v_{2}\right\rangle}{\operatorname{det}\left(v_{1}, v_{2}\right)}\right)\right],
$$

which is indeed regular at $\xi=0$, and, again,

$$
\mu^{L+\operatorname{lin}\left(\mathfrak{f}_{2}\right) / \operatorname{lin}\left(\mathfrak{f}_{2}\right)}\left(\left(\mathfrak{t}\left(\mathfrak{a}, \mathfrak{f}_{2}\right)\right)=0 .\right.
$$

\section{Barvinok valuations}

Let $\mathcal{L}$ be a finite family of rational vector subspaces of $V$, and let $\rho(L), L \in \mathcal{L}$, be a set of rational coefficients. The linear combination $\sum_{L \in \mathcal{L}} \rho(L) S^{L}(\mathfrak{p})$ is again a valuation on the set of polyhedra $\mathfrak{p} \subset V$, with values in $\mathcal{M}_{\ell}\left(V^{*}\right)$. By taking linear combinations, we obtain a local Euler-Maclaurin expansion for the function $\sum_{L \in \mathcal{L}} \rho(L) S^{L}(\mathfrak{p})(\xi)$.

Definition 12 Let $\mathfrak{p} \subset V$ be a polyhedron.

(a) We denote

$$
S^{(\mathcal{L}, \rho)}(\mathfrak{p})=\sum_{L \in \mathcal{L}} \rho(L) S^{L}(\mathfrak{p}) .
$$

(b) We define the $\mathfrak{f}$-term in the local Euler-Maclaurin expansion of $S^{(\mathcal{L}, \rho)}(\mathfrak{p})$ to be

$$
\mu^{(\mathcal{L}, \rho)}(\mathfrak{t}(\mathfrak{p}, \mathfrak{f}))=\sum_{L \in \mathcal{L}} \rho_{\mathcal{L}}(L) \mu^{L+\operatorname{lin}(\mathfrak{f}) / \operatorname{lin}(\mathfrak{f})}(\mathfrak{t}(\mathfrak{p}, \mathfrak{f}))
$$


Thus we have

$$
S^{(\mathcal{L}, \rho)}(\mathfrak{p})=\sum_{\mathfrak{f} \in \mathcal{F}(\mathfrak{p})} \mu^{(\mathcal{L}, \rho)}(\mathfrak{t}(\mathfrak{p}, \mathfrak{f})) I(\mathfrak{f}) .
$$

We are going to compute the $\mathfrak{f}$-term in the case of the following particular linear combinations introduced by Barvinok [2].

Definition 13 The valuation $S^{(\mathcal{L}, \rho)}$ is called a Barvinok valuation if

(a) the family of subspaces $\mathcal{L}$ is stable under sum,

(b) $\rho$ is an integer valued function on the set $\mathcal{L}$ such that the characteristic function of the union of the subspaces $L^{\perp} \subseteq V^{*}$ can be written as the linear combination

$$
\chi\left(\cup_{L \in \mathcal{L}} L^{\perp}\right)=\sum_{L \in \mathcal{L}} \rho(L) \chi\left(L^{\perp}\right) .
$$

Definition 14 We call a function $\mathcal{L} \rightarrow \mathbb{Z}$ which satisfies (8) a patchwork function on $\mathcal{L}$.

As the set of orthogonal subspaces $L^{\perp} \subseteq V^{*}$ is stable under intersection, a particular function $\rho_{\mathcal{L}}$ with this property can be computed in terms of the Moebius function of the partially ordered set $\mathcal{L}([7]$, vol I, section 3.7), as explained in [2].

Let us compute a patchwork function $\rho$ in the following case. $V=\mathbb{R}^{d}$ with standard basis $e_{i}, i=1, \ldots, d$, and $\mathcal{L}_{d, q}$ is the set of subspaces $L_{I}=\oplus_{i \in I} \mathbb{R} e_{i}$ with cardinal $|I| \geq q$. The function $\rho_{d, q}$ defined below is actually the one associated to the Moebius function, but we will not need this fact.

We denote the binomial coefficient $\frac{m !}{k !(m-k) !}$ by $C_{m}^{k}$.

Lemma 15 The function $\rho_{d, q}$ on $\mathcal{L}_{d, q}$ defined by

$$
\rho_{d, q}\left(L_{I}\right)=(-1)^{n-q} C_{n-1}^{q-1} \quad \text { if }|I|=n,
$$

satisfies Equation (8).

Proof. If $e^{i}$ is the dual basis, the orthogonal space $L_{I}^{\perp}$ is equal to $\sum_{i \notin I} \mathbb{R} e^{i}$. Let $\xi=\sum_{i=1}^{d} \xi_{i} e^{i} \in \cup_{L \in \mathcal{L}_{q}} L^{\perp}$. Let $I_{0}$ be the set of indices $i \in[1, \ldots, d]$ such that $\xi_{i}=0$. Then $\left|I_{0}\right| \geq q$, and $\xi \in L_{I}^{\perp}$ if and only $I \subseteq I_{0}$. Let $\left|I_{0}\right|=N$. The value at $\xi$ of the right-hand side of (8) is equal to

$$
E(N, q)=\sum_{I \subseteq I_{0}} \rho_{d, q}\left(L_{I}\right)=\sum_{n=q}^{N}(-1)^{n-q} C_{N}^{n} C_{n-1}^{q-1} .
$$


We want to prove that $E(N, q)=1$. Writing $n=q+i$, we have

$$
E(N, q)=\sum_{i=0}^{N-q}(-1)^{i} \frac{N !}{(q+i)(N-q-i) ! i !(q-1) !} .
$$

Let us compute $(q-1) !(E(N+1, q)-E(N, q))$. This is equal to

$$
\begin{gathered}
(-1)^{N+1-q} \frac{(N+1) !}{(N+1)(N+1-q) !}+\sum_{i=0}^{N-q}(-1)^{i} \frac{1}{(q+i) i !}\left(\frac{(N+1) !}{(N+1-q-i) !}-\frac{N !}{(N-q-i) !}\right) \\
=N ! \sum_{i=0}^{N+1-q}(-1)^{i} \frac{N !}{i !(N+1-q-i) !}=0 .
\end{gathered}
$$

We obtain $E(N, q)=E(q, q)=1$.

In the case of a Barvinok valuation, it turns out that the $\mathrm{f}$-term in the Euler-Maclaurin expansion of $S^{(\mathcal{L}, \rho)}(\mathfrak{p})$ coincides with that of $S(\mathfrak{p})$, if the vector subspace $\operatorname{lin}(\mathfrak{f})$ belongs to the set $\mathcal{L}$. This is the crucial result of the present article. It is an easy consequence of the following combinatorial lemma.

Lemma 16 Let $\mathcal{L}$ be a finite family of vector subspaces of $V$, stable under sum and let $\rho$ be a patchwork function on $\mathcal{L}$.

(a) Let $L_{0} \in \mathcal{L}$. Then

$$
\sum_{\left\{L \in \mathcal{L}, L \subseteq L_{0}\right\}} \rho(L)=1 .
$$

(b) Let $L_{0} \varsubsetneqq L_{1}$ be two subspaces in the family $\mathcal{L}$. Then

$$
\sum_{\left\{L \in \mathcal{L}, L+L_{0}=L_{1}\right\}} \rho(L)=0
$$

Proof. There exists a $\xi_{0} \in L_{0}^{\perp}$ such that, for $L \in \mathcal{L}, \xi_{0} \in L^{\perp}$ if and only if $L \subseteq L_{0}$. We obtain (a) by evaluating both sides of (8) at this particular element $\xi_{0}$. Next, we deduce (b) from (a), by induction on $\operatorname{dim} L_{1}-\operatorname{dim} L_{0}$.

For two subspaces $M \subseteq M^{\prime}$ in the family $\mathcal{L}$, let us denote

$$
f\left(M, M^{\prime}\right)=\sum_{\left\{L \in \mathcal{L}, L+M=M^{\prime}\right\}} \rho(L) .
$$


If $M=M^{\prime}$, we have $f(M, M)=\sum_{\{L \in \mathcal{L}, L \subseteq M\}} \rho(L)=1$ by $(a)$.

We apply this with $M=L_{1}$. Thus

$$
\sum_{\left\{L \in \mathcal{L}, L \subseteq L_{1}\right\}} \rho(L)=1
$$

In this sum, we group the $L \in \mathcal{L}$ such that $L+L_{0}$ is equal to a given $M_{1} \in \mathcal{L}$ together.

First we consider the case when $\operatorname{dim} L_{1}-\operatorname{dim} L_{0}=1$. Then $M_{1}$ is either $L_{0}$ or $L_{1}$, hence we obtain

$$
f\left(L_{0}, L_{0}\right)+f\left(L_{0}, L_{1}\right)=1
$$

Since $f\left(L_{0}, L_{0}\right)=1$ by $(a)$, we obtain $f\left(L_{0}, L_{1}\right)=0$ as required.

Next we consider the case when $\operatorname{dim} L_{1}-\operatorname{dim} L_{0}>1$. We obtain

$$
\sum_{\left\{M_{1} \in \mathcal{L}, L_{0} \subseteq M_{1} \subseteq L_{1}\right\}} f\left(L_{0}, M_{1}\right)=1 .
$$

For $M_{1}=L_{0}$, we have $f\left(L_{0}, L_{0}\right)=1$ by $(a)$. For $M_{1} \varsubsetneqq L_{1}$, we have $f\left(L_{0}, M_{1}\right)=0$ by induction hypothesis. Hence there remains only the term $f\left(L_{0}, L_{1}\right)$ which must be equal to 0 .

We study now the Euler-Maclaurin expansion of a Barvinok valuation.

Theorem 17 Let $\mathfrak{p} \subset V$ be a rational polyhedron and let $\mathfrak{f}$ be a face of $\mathfrak{p}$. Let $\mathcal{L}$ be a finite family of rational vector subspaces of $V$, stable under sum. Let $\rho$ be a patchwork function on $\mathcal{L}$, and let $S^{(\mathcal{L}, \rho)}=\sum_{L \in \mathcal{L}} \rho(L) S^{L}$.

Assume that $\operatorname{lin}(\mathfrak{f})$ belongs to $\mathcal{L}$. Then

$$
\mu^{(\mathcal{L}, \rho)}(\mathfrak{t}(\mathfrak{p}, \mathfrak{f}))=\mu^{\{0\}}(\mathfrak{t}(\mathfrak{p}, \mathfrak{f})) .
$$

In other words, the $\mathfrak{f}$-term in the local Euler-Maclaurin expansion of $S^{(\mathcal{L}, \rho)}(\mathfrak{p})$ coincides with that of $S(\mathfrak{p})$.

Proof. In the sum $\sum_{L \in \mathcal{L}} \rho(L) \mu^{L+\operatorname{lin}(\mathfrak{f}) / \operatorname{lin}(\mathfrak{f})}(\mathfrak{t}(\mathfrak{p}, \mathfrak{f}))$, we group the terms for which $L+\operatorname{lin}(\mathfrak{f})$ is equal to a given $L_{1}$ together. By Lemma 16 we obtain $\mu^{\{0\}}(\mathfrak{t}(\mathfrak{p}, \mathfrak{f}))$ for $L_{1}=\operatorname{lin}(\mathfrak{f})$ and 0 otherwise. 
Corollary 18 Let $\mathfrak{p} \subset V$ be a rational polyhedron. Let $0 \leq k \leq d$. Let $\mathcal{L}$ be a finite family of rational vector subspaces of $V$, stable under sum, such that $\operatorname{lin}(\mathfrak{f}) \in \mathcal{L}$ for every $k$-dimensional face $\mathfrak{f}$ of $\mathfrak{p}$ and let $\rho$ be a patchwork function on $\mathcal{L}$.

- Let $0<k \leq d$. Then the meromorphic function

$$
S(\mathfrak{p})(\xi)-S^{(\mathcal{L}, \rho)}(\mathfrak{p})(\xi)
$$

has lowest degree $\geq-k+1$.

- Let $k=0$. Then

$$
S(\mathfrak{p})(\xi)=S^{(\mathcal{L}, \rho)}(\mathfrak{p})(\xi) .
$$

Proof. By Theorem 17, the local Euler-Maclaurin expansion of the difference involves only faces of dimension $<k$.

$$
S(\mathfrak{p})(\xi)-S^{(\mathcal{L}, \rho)}(\mathfrak{p})(\xi)=\sum_{\{\mathfrak{f} \in \mathcal{F}(\mathfrak{p}), \operatorname{dim} \mathfrak{f}<k\}}\left(\mu^{\{0\}}(\mathfrak{t}(\mathfrak{p}, \mathfrak{f}))(\xi)-\mu^{(\mathcal{L}, \rho)}(\mathfrak{t}(\mathfrak{p}, \mathfrak{f}))(\xi)\right) I(\mathfrak{f})(\xi) .
$$

For a face of dimension $j$, the function $I(\mathfrak{f})(\xi)$ is homogeneous of degree $-j$. Multiplied by the holomorphic function $\mu^{\{0\}}(\mathfrak{t}(\mathfrak{p}, \mathfrak{f}))(\xi)-\mu^{\mathcal{L}}(\mathfrak{t}(\mathfrak{p}, \mathfrak{f}))(\xi)$, its lowest degree can only increase.

Remark that the statement of Corollary 18 above does not involve any scalar product. In the next section, we will show that this corollary implies our main Theorem (Theorem 20).

\section{Ehrhart quasi-polynomial}

Let $\mathfrak{p}$ be a rational polytope and let $h(x)$ be a polynomial function on $V$. Let

$$
S(\mathfrak{p}, h)=\sum_{x \in \mathfrak{p} \cap \mathbb{Z}^{d}} h(x)
$$

When $\mathfrak{p}$ is dilated by a non negative integer $n$, we obtain the Ehrhart quasipolynomial of the pair $(\mathfrak{p}, h)$

$$
S(n \mathfrak{p}, h)=\sum_{m=0}^{d+N} E_{m}(\mathfrak{p}, h, n) n^{m}
$$


where $N=\operatorname{deg} h$. The coefficients $E_{m}(\mathfrak{p}, h, n)$ are periodic functions of $n \in \mathbb{N}$, with period the smallest integer $q$ such that $q \mathfrak{p}$ is a lattice polytope.

If an integer $r \leq d$ is fixed, and $h=1$, Barvinok [2] proved that the $r+1$ highest Ehrhart coefficients $E_{d}(\mathfrak{p}, 1, n), \ldots, E_{d-r}(\mathfrak{p}, 1, n)$ of $S(n \mathfrak{p}, 1)$ can be computed in polynomial time with respect to $d$, when $\mathfrak{p}$ is a rational simplex.

Let $L \subseteq V$ be a rational vector subspace. Denote the projected lattice on $V / L$ by $\Lambda_{L}$. Consider the mixed valuation

$$
S^{L}(\mathfrak{p}, h)=\sum_{y \in \Lambda_{V / L}} \int_{\mathfrak{p} \cap(y+L)} h(x) d x .
$$

As shown by Barvinok, and as we will see here, we can use linear combination of these mixed valuations to approximate $S(n \mathfrak{p}, h)$ when $n$ is big.

For any polyhedron $\mathfrak{a}$, we define the meromorphic function $S^{L}(\mathfrak{a}, h)(\xi) \in$ $\mathcal{M}_{\ell}\left(V^{*}\right)$ similarly to $S^{L}(\mathfrak{a}, h)$. For $\xi \in V^{*}$ such that the sum converges, we have

$$
S^{L}(\mathfrak{a}, h)(\xi)=\sum_{y \in \Lambda_{V / L}} \int_{\mathfrak{a} \cap(y+L)} h(x) e^{\langle\xi, x\rangle} d m_{L}(x) .
$$

Remark 19 It is clear that $S^{L}(\mathfrak{a}, h)(\xi)=h\left(\partial_{\xi}\right) \cdot S^{L}(\mathfrak{a})(\xi)$.

If $\mathfrak{p}$ is a polytope, then $S^{L}(\mathfrak{p}, h)(\xi)$ is regular at $\xi=0$ and $S^{L}(\mathfrak{p}, h)(0)=$ $S^{L}(\mathfrak{p}, h)$.

For a family $\mathcal{L}$ and a function $L \mapsto \rho(L), L \in \mathcal{L}$, we define

$$
S^{(\mathcal{L}, \rho)}(\mathfrak{a}, h)(\xi)=\sum_{L \in \mathcal{L}} \rho(L) S^{L}(\mathfrak{a}, h)(\xi) .
$$

If $\mathfrak{p}$ is a polytope, and we dilate by $n \in N$, we have again a quasipolynomial

$$
S^{(\mathcal{L}, \rho)}(n \mathfrak{p}, h)(0)=\sum_{m=0}^{d+N} E_{m}(\mathcal{L}, \rho, \mathfrak{p}, h, n) n^{m} .
$$

We can replace the quasi-polynomial $S^{(\mathcal{L}, \rho)}(n \mathfrak{p}, h)(0)$ by $q$ legal polynomials in the variable $u$, by splitting $\mathbb{N}$ into classes modulo $q$. Writing $n=q u+k$, for $k=0, \ldots, q-1$, we obtain the polynomial function of $u$ :

$$
S^{(\mathcal{L}, \rho)}((q u+k) \mathfrak{p}, h)(0)=\sum_{m=0}^{d+N} E_{m}^{(k)}(\mathcal{L}, \rho, \mathfrak{p}, h) u^{m} .
$$


We briefly recall how the usual Ehrhart quasi-polynomial of a polytope can be computed using Brion's theorem. We will then use a similar method in order to compute efficiently the $r+1$ highest coefficients only, using Barvinok valuations.

Let $\mathcal{V}(\mathfrak{p})$ be the set of vertices of $\mathfrak{p}$. For each vertex $s$, let $\mathfrak{c}_{s}$ be the cone of feasible directions of $\mathfrak{p}$ at $s$, so that the supporting cone at $s$ is $s+\mathfrak{c}_{s}$. By Brion's theorem [5], we have

$$
S(\mathfrak{p}, h)(\xi)=\sum_{s \in \mathcal{V}(\mathfrak{p})} S\left(s+\mathfrak{c}_{s}, h\right)(\xi) .
$$

Let $n \in \mathbb{N}$ and consider the dilated polytope $n \mathfrak{p}$. The supporting cone at the vertex $n s$ is $n s+\mathfrak{c}_{s}$. Let $q \in \mathbb{N}$ such that $q \mathfrak{p}$ is a lattice polytope and fix $k \in \mathbb{N}, 0 \leq k \leq q-1$. Let $n=q u+k$. As qus is an integral point, we have

$$
S\left((q u+k) s+\mathfrak{c}_{s}, h\right)(\xi)=e^{q u\langle\xi, s\rangle} S^{\mathcal{L}_{s}, \rho_{s}}\left(k s+\mathfrak{c}_{s}, h\right)(\xi) .
$$

Expanding in powers of $u$, we obtain

$$
S((q u+k) \mathfrak{p}, h)(\xi)=\sum_{m \geq 0} u^{m} \frac{q^{m}}{m !} \sum_{s \in \mathcal{V}(\mathfrak{p})}\langle\xi, s\rangle^{m} S\left(k s+\mathfrak{c}_{s}, h\right)(\xi) .
$$

It follows that for each $m$, the sum of meromorphic functions

$$
\frac{q^{m}}{m !} \sum_{s \in \mathcal{V}(\mathfrak{p})}\langle\xi, s\rangle^{m} S\left(k s+\mathfrak{c}_{s}, h\right)(\xi)
$$

is actually analytic. Its value at $\xi=0$ is obtained by taking the zero degree term. We obtain

$$
S((q u+k) \mathfrak{p}, h)(0)=\sum_{m \geq 0} E_{m}^{(k)}(\mathfrak{p}, h) u^{m}
$$

with

$$
E_{m}^{(k)}(\mathfrak{p}, h)=\frac{q^{m}}{m !} \sum_{s \in \mathcal{V}(\mathfrak{p})}\langle\xi, s\rangle^{m} S\left(k s+\mathfrak{c}_{s}, h\right)_{[-m]}(\xi) .
$$

The right-hand side of this relation, a priori a meromorphic function of $\xi$, is actually constant. Moreover, we have $S\left(k s+\mathfrak{c}_{s}, h\right)_{[-m]}(\xi)=0$ if $m>d+N$, hence the Ehrhart quasi-polynomial has degree $\leq d+N$. 
We apply now Brion's theorem to $S^{\mathcal{L}, \rho}(\mathfrak{p}, h)(\xi)$. We obtain

$$
S^{\mathcal{L}, \rho}(\mathfrak{p}, h)(\xi)=\sum_{s \in \mathcal{V}(\mathfrak{p})} S^{\mathcal{L}, \rho}\left(s+\mathfrak{c}_{s}, h\right)(\xi) .
$$

For reasons to be explained later on, instead of one family $\mathcal{L}$, we take a family of subspaces $\mathcal{L}_{s}$ for each vertex $s$. Let $\rho_{s}: \mathcal{L}_{s} \rightarrow \mathbb{Z}$ be a function on $\mathcal{L}_{s}$. We denote now by $(\mathcal{L}, \rho)$ the map $s \mapsto\left(\mathcal{L}_{s}, \rho_{s}\right)$.

We define:

$$
\mathcal{B}^{\mathcal{L}, \rho}(\mathfrak{p}, h)(\xi)=\sum_{s \in \mathcal{V}(\mathfrak{p})} S^{\mathcal{L}_{s}, \rho_{s}}\left(s+\mathfrak{c}_{s}, h\right)(\xi) .
$$

If the family does not depend on $s,\left(\mathcal{L}_{s}, \rho_{s}\right)=\left(\mathcal{L}_{0}, \rho_{0}\right)$ for every vertex $s$, then, by Brion's theorem, we have

$$
\mathcal{B}^{\mathcal{L}, \rho}(\mathfrak{p}, h)(\xi)=S^{\mathcal{L}_{0}, \rho_{0}}(\mathfrak{p}, h)(\xi) .
$$

We dilate (9). Let $n=q u+k$. We obtain

$$
\mathcal{B}^{\mathcal{L}, \rho}((q u+k) \mathfrak{p}, h)(\xi)=\sum_{s \in \mathcal{V}(\mathfrak{p})} e^{q u\langle\xi, s\rangle} S^{\mathcal{L}_{s}, \rho_{s}}\left(k s+\mathfrak{c}_{s}, h\right)(\xi) .
$$

Expanding in powers of $u$, we obtain

$$
\mathcal{B}^{\mathcal{L}, \rho}((q u+k) \mathfrak{p}, h)(\xi)=\sum_{m \geq 0} u^{m} E_{m}^{(k)}(\mathcal{L}, \rho, \mathfrak{p}, h)(\xi)
$$

with

$$
E_{m}^{(k)}(\mathcal{L}, \rho, \mathfrak{p}, h)(\xi)=\frac{q^{m}}{m !} \sum_{s \in \mathcal{V}(\mathfrak{p})}\langle\xi, s\rangle^{m} S^{\mathcal{L}_{s}, \rho_{s}}\left(k s+\mathfrak{c}_{s}, h\right)(\xi) .
$$

If the family does not depend on $s,\left(\mathcal{L}_{s}, \rho_{s}\right)=\left(\mathcal{L}_{0}, \rho_{0}\right)$ for all vertices, then $\mathcal{B}^{\mathcal{L}, \rho}(\mathfrak{p}, h)(\xi)=S^{\mathcal{L}_{0}, \rho_{0}}(\mathfrak{p}, h)(\xi)$ is analytic near $\xi=0$, and so are the coefficients (11).

On the contrary, if we take a different family $\mathcal{L}_{s}$ for each vertex $s$, the coefficient $E_{m}^{(k)}(\mathcal{L}, \rho, \mathfrak{p}, h)(\xi)$ of $u^{m}$ in $(10)$ is no longer analytic near $\xi=0$, in general. However, the meromorphic function $\xi \mapsto E_{m}^{(k)}(\mathcal{L}, \rho, \mathfrak{p}, h)(\xi)$ belongs to $\mathcal{M}_{\ell}\left(V^{*}\right)$, thus it has a term of degree 0 with respect to $\xi$, given by

$$
E_{m}^{(k)}(\mathcal{L}, \rho, \mathfrak{p}, h)_{[0]}(\xi)=\frac{q^{m}}{m !} \sum_{s \in \mathcal{V}(\mathfrak{p})}\langle\xi, s\rangle^{m} S^{\mathcal{L}_{s}, \rho_{s}}\left(k s+\mathfrak{c}_{s}, h\right)_{[-m]}(\xi)
$$


For a family $(\mathcal{L}, \rho)$ as described in the next theorem, it turns out that, for large $m$, this zero-degree part $E_{m}^{(k)}(\mathcal{L}, \rho, \mathfrak{p}, h)_{[0]}(\xi)$ is actually analytic, hence constant, and its value is equal to the $m$-th Ehrhart coefficient $E_{m}^{(k)}(\mathfrak{p}, h)$ of $S((k+q u) \mathfrak{p}, h)(0)$.

Theorem 20 Let $\mathfrak{p}$ be a rational polytope in a rational vector space of dimension d. For each vertex $s$ of the polytope $\mathfrak{p}$, let $\mathfrak{c}_{s}$ be the cone of feasible directions of $\mathfrak{p}$ at $s$, so that the supporting cone at $s$ is $s+\mathfrak{c}_{s}$. For each vertex $s$, let $\mathcal{L}_{s}$ be a finite family of rational vector subspaces of $V$, stable under sum, such that $\operatorname{lin}(\mathfrak{f})$ belongs to $\mathcal{L}_{s}$ for every face $\mathfrak{f}$ of codimension $r$ of the cone $\mathfrak{c}_{s}$, and let $\rho_{s}$ be a patchwork function on $\mathcal{L}_{s}$. Let $q \in \mathbb{N}$ such that $q \mathfrak{p}$ is a lattice polytope and fix $k \in \mathbb{N}, 0 \leq k \leq q-1$. Let $h(x)$ be a homogeneous polynomial of total degree $N$.

Then, for $m \geq d+N-r$, the zero-degree term $E_{m}^{(k)}(\mathcal{L}, \rho, \mathfrak{p}, h)_{[0]}(\xi)$ defined by (19) is regular near $\xi=0$, hence constant. Its value is the coefficient $E_{m}^{(k)}(\mathfrak{p}, h)$ of $u^{m}$ in the Ehrhart quasi-polynomial

$$
S((k+q u) \mathfrak{p}, h)(0)=\sum_{x \in((k+q u) \mathfrak{p}) \cap \Lambda} h(x)=\sum_{m=0}^{d+N} u^{m} E_{m}^{(k)}(\mathfrak{p}, h) .
$$

Proof. We first consider the case $h(x)=1$. We have, for every $m \geq 0$,

$$
E_{m}^{(k)}(\mathfrak{p}, 1)=\frac{q^{m}}{m !} \sum_{s \in \mathcal{V}(\mathfrak{p})}\langle\xi, s\rangle^{m} S\left(k s+\mathfrak{c}_{s}\right)_{[-m]}(\xi)
$$

where the right-hand side is actually a constant function of $\xi$. For $m>$ $d-r-1$, we have, by Corollary 18 ,

$$
S\left(k s+\mathfrak{c}_{s}\right)(\xi)_{[-m]}=S^{\mathcal{L}_{s}, \rho_{s}}\left(k s+\mathfrak{c}_{s}\right)_{[-m]}(\xi) .
$$

This proves the theorem when $h(x)=1$. The case of a non constant polynomial $h(x)$ is quite similar. If $h(x)=x_{1}^{N_{1}} \ldots x_{d}^{N_{d}}$, we just have to replace the meromorphic functions $S\left(k s+\mathfrak{c}_{s}\right)(\xi)$ and $S^{\mathcal{L}_{s}, \rho_{s}}\left(k s+\mathfrak{c}_{s}\right)(\xi)$ by their derivatives under $\partial_{\xi_{1}}^{N_{1}} \ldots \partial_{\xi_{d}}^{N_{d}}$.

If for each vertex $s$, we take $\mathcal{L}_{s}=\mathcal{L}$, the full collection generated by all $r$ codimensional faces of $\mathfrak{p}$, we obtain Corollary 21 below, that is Barvinok's theorem [2], with an extension to the sum of values of any polynomial $h(x)$ over the set of integral points of a rational polytope (Barvinok considers only the case $h(x)=1)$. 
Corollary 21 Let $\mathfrak{p} \subset V$ be a rational polytope and let $h(x)$ be a polynomial function on $V$. Let $\mathcal{L}$ be a finite family of rational vector subspaces of $V$, stable under sum. Assume that $\operatorname{lin}(\mathfrak{f})$ belongs to $\mathcal{L}$ for every face $\mathfrak{f}$ of codimension $r$ of $\mathfrak{p}$. Let $\rho$ be a patchwork function on $\mathcal{L}$ and let $S^{\mathcal{L}, \rho}=\sum_{L \in \mathcal{L}} \rho(L) S^{L}$. Then the $r+1$ highest Ehrhart coefficients of $S(t \mathfrak{p}, h)(0)$ and $S^{\mathcal{L}, \rho}(t \mathfrak{p}, h)(0)$ are equal.

The point in taking a family $\mathcal{L}_{s}$ which depends on the vertex $s$ lies in the case where $\mathfrak{p}$ is simplicial. In this case, we can take $\mathcal{L}_{s}$ to be just the set of subspaces $\operatorname{lin}(\mathfrak{f})$, for all faces $\mathfrak{f}$ of codimension $\leq r$ of the supporting cone $\mathfrak{c}_{s}$ at vertex $s$. This family is stable under sum. Moreover the patchwork function on $\mathcal{L}_{s}$ is simple, (Lemma 15) and the computation of the function $S^{L}\left(k s+\mathfrak{c}_{s}\right)(\xi)$, when $L \in \mathcal{L}_{s}$, is immediately reduced (Example 6) to the computation of a function $S(\mathfrak{a})(\xi)$ for a simplicial cone $\mathfrak{a}$ in a rational vector space of dimension smaller or equal than $r$. When $\mathfrak{p}$ is a simplex, we obtain in this way a method for computing the $r+1$ highest Ehrhart coefficients for the pair $(\mathfrak{p}, h)$.

\section{Local Euler-Maclaurin formula for mixed sums}

Finally in this last section, we discuss an application of the existence of the coefficients $\mu^{L}$ (Theorem 8) in the line of 4 .

Let $\mathfrak{p}$ be a rational polytope in a rational vector space $V$ of dimension $d$ and let $h(x)$ be a polynomial function on $V$. Let $L$ be a rational subspace of $V$. Consider the mixed sum

$$
S^{L}(\mathfrak{p}, h)=\sum_{y \in \Lambda_{V / L}} \int_{\mathfrak{p} \cap(y+L)} h(x) d m_{L}(x) .
$$

As in [4], we associate to the analytic function $\mu^{L}(\mathfrak{t}(\mathfrak{p}, \mathfrak{f}))$ a constant coefficients differential operator (of infinite order) on $V$.

Definition 22 Let $\mathfrak{f}$ be a face of $\mathfrak{p}$. We denote by $D^{L}(\mathfrak{p}, \mathfrak{f})$ the differential operator on $V$ associated to analytic function $\mu^{L}(\mathfrak{t}(\mathfrak{p}, \mathfrak{f}))$ :

$$
\left.D^{L}(\mathfrak{p}, \mathfrak{f})\left(\partial_{\xi}\right) \cdot e^{\langle\xi, x\rangle}=\mu^{L}(\mathfrak{t}(\mathfrak{p}, \mathfrak{f}))\right)(\xi) e^{\langle\xi, x\rangle}
$$


The operators $D^{L}(\mathfrak{p}, \mathfrak{f})$ are local, that is they depend only of the transverse cone $\mathfrak{t}(\mathfrak{p}, \mathfrak{f})$ of $\mathfrak{p}$ along $\mathfrak{f}$, and they involve only derivatives in directions orthogonal to the face $\mathfrak{f}$. We can state the following theorem with the same proof as in [4].

Theorem 23 (Local Euler-Maclaurin formula) Let $\mathfrak{p}$ be a polytope in $V$. For any polynomial function $h(x)$ on $V$, we have

$$
S^{L}(\mathfrak{p}, h)=\sum_{\mathfrak{f} \in \mathcal{F}(\mathfrak{p})} \int_{\mathfrak{f}} D^{L}(\mathfrak{p}, \mathfrak{f}) \cdot h
$$

where the integral on the face $\mathfrak{f}$ is taken with respect to the Lebesgue measure on $\langle\mathfrak{f}\rangle$ defined by the lattice $\Lambda \cap \operatorname{lin}(\mathfrak{f})$.

In particular, for $h=1$, we obtain

$$
S^{L}(\mathfrak{p}, 1)=\sum_{\mathfrak{f} \in \mathcal{F}(\mathfrak{p})} \mu^{L}(\mathfrak{t}(\mathfrak{p}, \mathfrak{f}))(0) \operatorname{vol}(\mathfrak{f}) .
$$

Let us dilate the polytope $\mathfrak{p}$ by a non negative integer $n$. If $\mathfrak{f}$ is a face of $\mathfrak{p}$, let $q_{\mathfrak{f}}$ be the smallest positive integer such that $q_{\mathfrak{f}}<\mathfrak{f}>$ contains integral points. Define $D(\mathfrak{p}, \mathfrak{f}, n)=D(n \mathfrak{p}, n \mathfrak{f})$, if $n>0$, and $D(\mathfrak{p}, \mathfrak{f}, 0)=D\left(q_{\mathfrak{f}} \mathfrak{p}, q_{\mathfrak{f}} \mathfrak{f}\right)$. The function $n \mapsto D(\mathfrak{p}, \mathfrak{f}, n)$ is periodic of period $q_{\mathfrak{f}}$.

Proposition 24 Let $\mathfrak{p}$ be a rational polytope and $h$ a polynomial function of degree $N$ on $V$. Then, for any integer $n \geq 0$, we have

$$
S^{L}(n \mathfrak{p}, h)=\sum_{\mathfrak{f} \in \mathcal{F}(\mathfrak{p})} \int_{n \mathfrak{f}} D^{L}(\mathfrak{p}, \mathfrak{f}, n) \cdot h .
$$

Furthermore, if $\mathfrak{f} \in \mathcal{F}(\mathfrak{p})$, we have

$$
\int_{n \mathfrak{f}} D^{L}(\mathfrak{p}, \mathfrak{f}, n) \cdot h=\sum_{i=\operatorname{dim} \mathfrak{f}}^{\operatorname{dim} \mathfrak{f}+N} E_{i}(\mathfrak{p}, h, \mathfrak{f}, n) n^{i}
$$

where the coefficients $E_{i}(\mathfrak{p}, h, \mathfrak{f}, n)$ are periodic with period $q_{\mathfrak{f}}$.

Hence the Ehrhart coefficients are given by

$$
E_{m}^{L}(\mathfrak{p}, h, n)=\sum_{\mathfrak{f}, \operatorname{dim} \mathfrak{f} \leq m} E_{m}(\mathfrak{p}, h, \mathfrak{f}, n) .
$$


When we apply the last proposition to the function $h(x)=1$, we obtain

$$
S^{L}(n \mathfrak{p}, 1)=\sum_{\mathfrak{f} \in \mathcal{F}(\mathfrak{p})} \mu^{L}(n \mathfrak{t}(\mathfrak{p}, \mathfrak{f}))(0) \operatorname{vol}(\mathfrak{f}) n^{\operatorname{dim} \mathfrak{f}}
$$

As $\mu^{L}(n \mathfrak{t}(\mathfrak{p}, \mathfrak{f}))$ is invariant by integral translations, the function $\mu^{L}(n \mathfrak{t}(\mathfrak{p}, \mathfrak{f}))(0)$ is of period $q_{\mathfrak{f}}$.

\section{References}

[1] Barvinok A. I.,Computing the Ehrhart polynomial of a convex lattice polytope, Discrete Comput. geom. 12 (1994), 35-48.

[2] Barvinok A. I.,Computing the Ehrhart quasi-polynomial of a rational simplex, Mathematics of Computation, 75 (2006), 1449-1466.

[3] Barvinok A. I. and Pommersheim J., An algorithmic theory of lattice points in polyhedra, New Perspectives in Algebraic Combinatorics (Berkeley,CA, 1996-97), Math. Sci. Res. Inst. Public 38, Cambridge University Press, Cambridge, (1999), pp 91-147.

[4] Berline N. and Vergne M. Local Euler-Maclaurin formula for polytopes (2005), arXiv:math CO/0507256. To appear in Moscow Math. J.

[5] Brion M., Points entiers dans les polyèdres convexes, Ann. Sci. Ecole Norm. Sup. 21 (1988), 653-663.

[6] De Loera J.A., Haws D., Hemmecke R., Huggins H., Tauzer J. and Yoshida R., A Users Guide for LattE v1.1, 2003, software package LattE, available at http://www.math.ucdavis.edu/ latte.

[7] Stanley R. Enumerative combinatorics. Vol 1 (1997), Cambridge Studies in Advanced Math. 49.

Acknowledgments: We thank the various institutions that helped us to collaborate on this work: the Research-in-pairs program at the Forschungsinstitut Oberwolfach, the University Denis Diderot in Paris and the Centre Laurent Schwartz at Ecole Polytechnique.

Velleda Baldoni, Universita di Roma Tor Vergata, Dipartimento di Matematica, via della Ricerca Scientifica, 00133 Roma, Italy. 
email: baldoni@mat.uniroma2.it

Nicole Berline, Ecole Polytechnique, Centre de mathématiques Laurent Schwartz, 91128, Palaiseau, France.

email: berline@math.polytechnique.fr

Michèle Vergne, Institut de Mathématiques de Jussieu, Théorie des Groupes, Case 7012, 2 Place Jussieu, 75251 Paris Cedex 05, France;

Ecole Polytechnique, Centre de mathématiques Laurent Schwartz, 91128, Palaiseau, France.

email: vergne@math.polytechnique.fr 